\title{
Power Allocation for Error Covariance Minimization in Kalman Filtering Over Packet Dropping Links
}

\author{
Alex S. Leong and Subhrakanti Dey
}

\begin{abstract}
This paper studies the problem of optimal power allocation for Kalman filtering with random packet losses. We consider the problem of minimizing the trace of the expected error covariance subject to an average transmit power constraint. Adopting a model for the packet loss probabilities that depends on both the sensor transmit power and timevarying fading channel gain, the problem is formulated as a constrained Markov decision process (MDP) that is solved numerically with dynamic programming techniques. Simpler suboptimal power allocation policies, namely a constant power allocation scheme and a truncated channel inversion scheme, are also considered and numerical comparisons made with the optimal policy.
\end{abstract}

\section{INTRODUCTION}

The problem of Kalman filtering with random packet losses has been studied extensively since the seminal work of [1], which showed that for i.i.d. Bernoulli packet losses there exists a critical threshold such that if the packet arrival rate exceeds this threshold the expected error covariance remains bounded, but diverges otherwise. This work has been extended in various directions such as: multiple sensors [2], [3], further characterizations of the critical threshold [4], [5], probabilistic notions of performance [6], [7], performing local processing before transmission [8], consideration of delays [9], and Markovian packet losses [10]-[12]. Kalman filtering over continuous valued fading channels has also been considered in [13], [14].

In the wireless communications community, power control is regularly used to improve system performance and reliability. The primary focus of the previously mentioned works is on deriving conditions for stability of the estimator, and power control is not explicitly considered. However, power control can also be used in Kalman filtering to improve the estimator stability and estimation performance. For Kalman filtering over continuous fading channels, the use of power control for outage minimization and expected error covariance minimization has been studied in [15]. The works of [16], [17] consider the use of power control at the sensor over a continuous fading channel, with the data being sent over this channel after digital modulation, which would then give a corresponding packet loss probability dependent on the transmit power at the sensor. Power allocation using model predictive control techniques is considered in [16], while optimal power allocation schemes to guarantee stability are investigated in [17].

This work was supported by the Australian Research Council.

The authors are with the Department of Electrical and Electronic Engineering, University of Melbourne, Vic. 3010, Australia, $\{$ asleong, sdey\}@unimelb.edu.au
In this paper we adopt a similar channel model to [17], but instead of using power allocation to achieve filter stability, we are interested in the use of power allocation to improve the estimation performance of the Kalman filter. Specifically, we address the problem of minimizing the trace of the expected error covariance subject to an average transmit power constraint. The problem is formulated as a Markov Decision Process (MDP) problem that can be solved numerically with dynamic programming techniques. Two simpler suboptimal schemes will also be investigated, namely a constant power allocation scheme, and a truncated channel inversion scheme, and their performance is compared with that of the optimal policy.

The paper is organized as follows. The system model is given in Section II. The optimal power allocation problem is formulated and solved in Section III, while suboptimal approaches are considered in Section IV. Numerical studies comparing the optimal and suboptimal schemes are given in Section V.

\section{SYSTEM MODEL}

A diagram of the system model is given in Fig. 1. Consider a linear system

$$
x_{k+1}=A x_{k}+w_{k}
$$

where $x_{k} \in \mathbb{R}^{n}$, and $w_{k}$ is i.i.d. Gaussian with zero mean and covariance matrix $Q>0 .{ }^{1}$ The sensor makes a measurement

$$
y_{k}=C x_{k}+v_{k}
$$

where $y_{k} \in \mathbb{R}^{m}$, and $v_{k}$ is i.i.d. Gaussian with zero mean and covariance matrix $R \geq 0$. We consider unstable systems, and assume that the pair $\left(A, Q^{1 / 2}\right)$ is stabilizable and the pair $(A, C)$ is detectable.

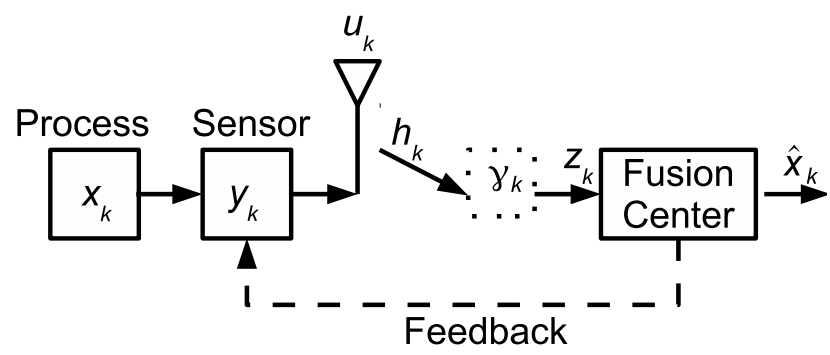

Fig. 1. System model

\footnotetext{
${ }^{1}$ We say that a matrix $X>0$ if $X$ is positive definite, and $X \geq 0$ if $X$ is positive semi-definite.
} 
The measurement is then sent to a fusion center (that performs the Kalman filtering operation) over a packet dropping link, which can be modelled by

$$
z_{k}=\gamma_{k} y_{k}
$$

where $z_{k}$ is the quantity received at the fusion center. Here the measurement $y_{k}$ is assumed to be encoded to form a single packet, and $\gamma_{k}=1$ denotes that the measurement packet is received, while $\gamma_{k}=0$ denotes that the packet is lost.

The Kalman filter state estimates and error covariances are defined as

$$
\begin{aligned}
& \hat{x}_{k \mid k}= \mathbb{E}\left[x_{k} \mid z_{0}, \ldots, z_{k}, \gamma_{0}, \ldots, \gamma_{k}\right] \\
& \hat{x}_{k+1 \mid k}=\mathbb{E}\left[x_{k+1} \mid z_{0}, \ldots, z_{k}, \gamma_{0}, \ldots, \gamma_{k}\right] \\
& P_{k \mid k}=\mathbb{E}\left[\left(x_{k}-\hat{x}_{k \mid k}\right)\left(x_{k}-\hat{x}_{k \mid k}\right)^{T} \mid z_{0}, \ldots, z_{k}, \gamma_{0}, \ldots, \gamma_{k}\right] \\
& P_{k+1 \mid k}=\mathbb{E}\left[\left(x_{k+1}-\hat{x}_{k+1 \mid k}\right)\left(x_{k+1}-\hat{x}_{k+1 \mid k}\right)^{T} \mid\right. \\
&\left.z_{0}, \ldots, z_{k}, \gamma_{0}, \ldots, \gamma_{k}\right] .
\end{aligned}
$$

We will also use the shorthand $P_{k+1} \triangleq P_{k+1 \mid k}$. The Kalman filtering equations with packet loss can be found in [1]. In particular, the error covariance $P_{k}$ satisfies

$$
P_{k+1}=A P_{k} A^{T}+Q-\gamma_{k} A P_{k} C^{T}\left(C P_{k} C^{T}+R\right)^{-1} C P_{k} A^{T} .
$$

Similar to [17], we adopt a model for the packet loss process $\left\{\gamma_{k}\right\}$ that is governed by a time-varying wireless fading channel $\left\{h_{k}\right\}$ and sensor transmit power control $\left\{u_{k}\right\}$ over this channel. In this model, the conditional packet reception probabilities are given by

$$
\operatorname{Pr}\left(\gamma_{k}=1 \mid u_{k}, h_{k}\right) \triangleq f\left(h_{k} u_{k}\right)
$$

where $f():.[0, \infty) \rightarrow[0,1]$ is a monotonically increasing continuous function. The form of $f($.$) will depend on the$ particular digital modulation scheme being used [18], see e.g. (8) for the case of binary phase shift keying (BPSK) transmission.

We consider the case where $\left\{h_{k}\right\}$ is a i.i.d. block fading process [19], where the channel remains constant over a fading block (representing the coherence time of the channel [20]) but can vary from block to block in an i.i.d. manner. ${ }^{2}$ We assume that channel state information (CSI) is available at the receiver/fusion center such that the fusion center knows the values of the channel gains $h_{k}$ at time $k{ }^{3}$ Since CSI is assumed to be available, we will allow the sensor transmit power $u_{k}$ to depend on both $h_{k}$ and $P_{k}$. In the next section we consider optimal power allocation to minimize the trace of the expected error covariance. Due to limited computational resources at the sensor, the optimal sensor transmit powers are usually computed at the fusion center and fed back to the sensor.

\footnotetext{
${ }^{2}$ Our formulation should also be able to be extended to other fading models such as the Markovian fading channel model of [21], which will be addressed in future work.

${ }^{3}$ In practice this can be achieved by periodically sending pilot signals either from the sensor to the fusion center to allow the fusion center to estimate the channel, or from the fusion center to the sensor under channel reciprocity (such as in time-division duplex channels).
}

\section{OPTIMAL POWER ALLOCATION}

\section{A. Problem statement}

The problem we consider in this paper is to determine the optimal sensor transmit power allocation, in order to minimize the trace of the expected error covariance subject to an average transmit power constraint $\mathcal{P}$, i.e.

$$
\begin{aligned}
& \min _{\left\{u_{k}\right\}} \limsup _{K \rightarrow \infty} \frac{1}{K} \sum_{k=0}^{K-1} \mathbb{E}\left[\operatorname{tr}\left(P_{k+1}\right)\right] \\
& \text { s.t. } \limsup _{K \rightarrow \infty} \frac{1}{K} \sum_{k=0}^{K-1} \mathbb{E}\left[u_{k}\right] \leq \mathcal{P} .
\end{aligned}
$$

The solution to problem (2) will be given in the next subsection.

Remark 3.1: Since Kalman filtering with packet losses can have unbounded expected error covariances in certain situations such as in [1], this raises the question as to whether problem (2) is well-posed. In [17] the authors study the problem of determining the minimum average power required for guaranteeing that the following exponential boundedness condition for the expected error covariance is satisfied:

$$
\mathbb{E}\left\|P_{k}\right\| \leq a \rho^{k}+b, \forall k
$$

for some $a$ and $b$, where $\rho \in[0,1)$, and where the packet reception probabilities are given by (1). Choosing $\mathcal{P}$ in the average power constraint of problem (2) to be greater than this minimum average power (see [17] for details on how to compute this minimum average power) will be sufficient to make the problem (2) well-posed.

\section{B. Solution to optimal power allocation problem}

The optimization problem (2) can be regarded as a constrained average cost Markov Decision Process (MDP) [22] with $\left(P_{k}, h_{k}\right)$ as the "state" and $u_{k}$ as the "action" of the MDP. To solve this problem, we will use a Lagrangian technique similar to [15], [22], [23] that considers instead the following unconstrained MDP problem:

$$
\begin{aligned}
& \min _{\left\{u_{k}\right\}} \limsup _{K \rightarrow \infty} \frac{1}{K} \sum_{k=0}^{K-1} \mathbb{E}\left[\operatorname{tr}\left(P_{k+1}\right)+\beta u_{k}\right] \\
& =\min _{\left\{u_{k}\right\}} \limsup _{K \rightarrow \infty} \frac{1}{K} \sum_{k=0}^{K-1} \mathbb{E}\left[\mathbb{E}\left[\operatorname{tr}\left(P_{k+1}\right) \mid P_{k}, h_{k}, u_{k}\right]+\beta u_{k}\right]
\end{aligned}
$$

where $\beta \geq 0$ is a Lagrange multiplier that specifies the tradeoff between the average transmit power and expected error covariance. Solving (3) for different values of $\beta$ will correspond to minimizing the trace of the expected error covariance for different average transmit power constraints in problem (2).

Remark 3.2: Under additional assumptions such as Assumption 3.1 (see later), one can show the absence of a duality gap by verifying the conditions provided in [22]. 
The average cost optimality equation or Bellman equation associated with problem (3) can then be written as

$$
\begin{aligned}
\rho+ & H\left(P_{k}, h_{k}\right)=\min _{u_{k}}\left[\mathbb{E}\left[\operatorname{tr}\left(P_{k+1}\right) \mid P_{k}, h_{k}, u_{k}\right]+\beta u_{k}\right. \\
& \left.+\int_{h_{k+1}, P_{k+1}} H\left(P_{k+1}, h_{k+1}\right) F\left(d\left(P_{k+1}, h_{k+1}\right) \mid P_{k}, h_{k}, u_{k}\right)\right]
\end{aligned}
$$

where $\rho$ is the optimal average cost per stage, $H$ the differential cost, and $F$ the probability transition law.

We first show that there exists stationary solutions to the MDP (3). We will make the following additional assumption.

Assumption 3.1: The range of $u_{k}$ is bounded, i.e. $u_{k} \in$ $\left[0, u_{\max }\right], \forall k$.

This assumption is obviously justified from a practical point of view. Additionally, it will allow us to show the following result:

Lemma 1: Under Assumption 3.1, there exists a stationary solution to the Bellman equation (4) which solves the MDP (3).

Proof: The proof involves verifying for our problem (3)-(4) the conditions from [24] that guarantee the existence of stationary solutions for MDPs with Borel state and action spaces. The verification of these conditions is very similar to the proof of Lemma 3 in [15]. The details are omitted for brevity.

For computational purposes, the Bellman equation can be further simplified as follows:

$$
\begin{aligned}
\rho & +H\left(P_{k}, h_{k}\right)=\min _{u_{k}}\left[\mathbb{E}\left[\operatorname{tr}\left(P_{k+1}\right) \mid P_{k}, h_{k}, u_{k}\right]+\beta u_{k}\right. \\
& \left.+\int_{h_{k+1}, P_{k+1}} H\left(P_{k+1}, h_{k+1}\right) F\left(d\left(P_{k+1}, h_{k+1}\right) \mid P_{k}, h_{k}, u_{k}\right)\right] \\
& =\min _{u_{k}}\left\{\operatorname{tr}\left(A P_{k} A^{T}+Q\right)+\beta u_{k}\right. \\
& -f\left(h_{k} u_{k}\right) \operatorname{tr}\left(A P_{k} C^{T}\left(C P_{k} C^{T}+R\right)^{-1} C P_{k} A^{T}\right) \\
& \left.+\int_{P_{k+1}, h_{k+1}} H\left(P_{k+1}, h_{k+1}\right) F\left(d\left(P_{k+1}, h_{k+1}\right) \mid P_{k}, h_{k}, u_{k}\right)\right\} \\
& \stackrel{(a)}{=} \min _{u_{k}}\left\{\operatorname{tr}\left(A P_{k} A^{T}+Q\right)+\beta u_{k}\right. \\
& -f\left(h_{k} u_{k}\right) \operatorname{tr}\left(A P_{k} C^{T}\left(C P_{k} C^{T}+R\right)^{-1} C P_{k} A^{T}\right) \\
& \left.+\int_{P_{k+1}, h_{k+1}} H\left(P_{k+1}, h_{k+1}\right) F\left(d P_{k+1} \mid P_{k}, h_{k}, u_{k}\right) F\left(d h_{k+1}\right)\right\} \\
& \stackrel{(b)}{=} \min _{u_{k}}\left\{\operatorname{tr}\left(A P_{k} A^{T}+Q\right)+\beta u_{k}\right. \\
& -f\left(h_{k} u_{k}\right) \operatorname{tr}\left(A P_{k} C^{T}\left(C P_{k} C^{T}+R\right)^{-1} C P_{k} A^{T}\right) \\
& +\int_{h_{k+1}}\left[H\left(A P_{k} A^{T}+Q, h_{k+1}\right)\left(1-f\left(h_{k} u_{k}\right)\right)\right. \\
& +H\left(A P_{k} A^{T}+Q-A P_{k} C^{T}\left(C P_{k} C^{T}+R\right)^{-1} C P_{k} A^{T}, h_{k+1}\right) \\
& \left.\left.\times f\left(h_{k} u_{k}\right)\right] F\left(d h_{k+1}\right)\right\} \\
& +5)
\end{aligned}
$$

where (a) follows from the fact that $h_{k+1}$ is independent of $P_{k+1}$, and (b) follows from writing out the conditional expectation $\mathbb{E}\left[H\left(P_{k+1}, h_{k+1}\right) \mid P_{k}, h_{k}, u_{k}\right]$. For numerical implementation, a discretized version of the Bellman equation (5) can then be solved using e.g. the relative value iteration algorithm [25] to find solutions to the MDP (3).

Remark 3.3: The discretized solution is strictly speaking a suboptimal approximation to the true optimal solution, however the use of discretization is generally unavoidable for MDPs with continuous state and action spaces. As the number of discretization levels increases, one would expect the discretized solution to converge to the optimal solution [26].

Now let $P^{*}(u)$ be the minimum trace of the expected error covariance such that the average transmit power is less than $u$. By solving the MDP (3) for different values of $\beta$, one can obtain points of the function $P^{*}(u)$, corresponding to different tradeoffs between the average transmit power and trace of the expected error covariance, see e.g. Fig. 2 in the next section. We have the following characterization of the function $P^{*}(u)$ :

Lemma 2: Suppose $f($.$) is a strictly concave function.$ Then $P^{*}(u)$ is a decreasing strictly convex function of $u$.

Proof: See appendix.

An example of a strictly concave $f($.$) is given by (8) in$ Section V. Using Lemma 2, one can conclude from the theory of Pareto optimality that all points on the curve $P^{*}(u)$ can be obtained by solving the MDP (3) for an appropriate choice of $\beta$, see [27], [28] for further details.

\section{Suboptimal POLICIES}

The optimal solution considered in the previous section requires the solution of a MDP which is computationally demanding, particularly for vector systems. In this section we consider two suboptimal policies which are simpler to compute and implement than the optimal solution of Section III.

\section{A. Constant power allocation}

One very simple scheme is to use constant power allocation, where $u_{k}=u_{\text {const }}, \forall k$. With this policy, the conditional packet reception probabilities $f\left(h_{k} u_{\text {const }}\right)$ will depend on the channel gain $h_{k}$ only.

\section{B. Truncated channel inversion}

Another possible scheme is based on the concept of channel inversion, which is a simple but quite commonly used technique in wireless communications, that attempts to invert the channel at every time instance to maintain a constant quality of service. However, it is known that for certain fading distributions such as Rayleigh fading, channel inversion actually requires infinite average power, so some modifications to the scheme such as truncation (where channel inversion is only carried out if the channel gain is sufficiently large) is necessary [29]. The power allocation policy we consider here is of the following form:

$$
u_{k}=\left\{\begin{array}{ccc}
\frac{\alpha}{h_{k}} & , & h_{k}>h^{*} \\
\frac{\alpha}{h^{*}} & , & \text { otherwise }
\end{array}\right.
$$


where $\alpha$ and $h^{*}$ are values that can be chosen by us. This scheme inverts the channel $h_{k}$ and multiplies it by a gain $\alpha$ if $h_{k}$ is greater than some threshold $h^{*}$, otherwise it transmits with the constant power $\frac{\alpha}{h^{*}} \cdot{ }^{4}$ The average transmit power using this scheme is

$$
\begin{aligned}
\mathbb{E}\left[u_{k}\right] & =\int_{h^{*}}^{\infty} \frac{\alpha}{h_{k}} F\left(d h_{k}\right)+\int_{0}^{h^{*}} \frac{\alpha}{h^{*}} F\left(d h_{k}\right) \\
& =\alpha E\left(h^{*}\right)+\frac{\alpha}{h^{*}} F_{H}\left(h^{*}\right), \forall k
\end{aligned}
$$

where

$$
E\left(h^{*}\right) \triangleq \int_{h^{*}}^{\infty} \frac{1}{h_{k}} F\left(d h_{k}\right),
$$

and $F_{H}($.$) is the cumulative distribution function of h_{k}$. For instance, if $h_{k} \sim \exp (1)$ (which is an example of Rayleigh fading [20]), we have $E\left(h^{*}\right)=\int_{h^{*}}^{\infty} \exp \left(-h_{k}\right) / h_{k} d h_{k}=$ $E_{1}\left(h^{*}\right)$, the exponential integral, and $F_{H}\left(h^{*}\right)=1-$ $\exp \left(-h^{*}\right)$.

In terms of the packet loss process $\left\{\gamma_{k}\right\}$, under this power allocation scheme, $\gamma_{k}=1$ with probability $f(\alpha)$ when $h_{k}>$ $h^{*}$, and $\gamma_{k}=1$ with probability $f\left(\frac{\alpha h_{k}}{h^{*}}\right)$ when $h_{k} \leq h^{*}$. That is, we have

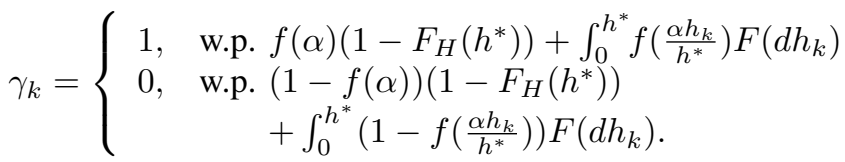

Therefore, using this scheme, $\gamma_{k}$ becomes an i.i.d. Bernoulli process with probability of packet reception $f(\alpha)(1-$ $\left.F_{H}\left(h^{*}\right)\right)+\int_{0}^{h^{*}} f\left(\frac{\alpha h_{k}}{h^{*}}\right) F\left(d h_{k}\right)$, and probability of packet loss $(1-f(\alpha))\left(1-F_{H}\left(h^{*}\right)\right)+\int_{0}^{h^{*}}\left(1-f\left(\frac{\alpha h_{k}}{h^{*}}\right)\right) F\left(d h_{k}\right)$.

As the values $\alpha$ and $h^{*}$ can be chosen by us, we can optimize $\alpha$ and $h^{*}$ to minimize the trace of the expected error covariance subject to an average power constraint, i.e. solving problem (2) but with $u_{k}$ restricted to be of the form (6). For Bernoulli packet losses it is known that the expected error covariance is a decreasing function of the packet reception probability [1]. Hence the problem is equivalent to minimizing the probability of packet loss subject to an average power constraint $\mathcal{P}$, i.e.

$$
\begin{aligned}
& \min _{\alpha, h^{*}}(1-f(\alpha))\left(1-F_{H}\left(h^{*}\right)\right)+\int_{0}^{h^{*}}\left(1-f\left(\frac{\alpha h_{k}}{h^{*}}\right)\right) F\left(d h_{k}\right) \\
& \text { s.t. } \alpha E\left(h^{*}\right)+\frac{\alpha}{h^{*}} F_{H}\left(h^{*}\right)=\mathcal{P} \text {. }
\end{aligned}
$$

We can further simplify problem (7) by rearranging the constraint to express $\alpha$ in terms of $h^{*}$, i.e.

$$
\alpha=\frac{\mathcal{P}}{E\left(h^{*}\right)+\frac{1}{h^{*}} F_{H}\left(h^{*}\right)} .
$$

The optimization problem (7) then becomes a onedimensional line search over $h^{*}$ that can be easily solved numerically.

\footnotetext{
${ }^{4}$ This scheme is essentially equivalent to the saturated constant gain power control considered in [17], but with different notations.
}

\section{NUMERICAL STUdies}

We present here numerical results for a scalar system with parameters $A=1.2, C=1, Q=1, R=1$. We consider a model for the packet loss probabilities studied in [17]. In this model we assume that the digital communication uses binary phase shift keying (BPSK) transmission [18] with $b$ bits per packet, so that we have

$$
\operatorname{Pr}\left(\gamma_{k}=1 \mid h_{k}, u_{k}\right)=f\left(h_{k} u_{k}\right)=\left(\int_{-\infty}^{\sqrt{h_{k} u_{k}}} \frac{1}{\sqrt{2 \pi}} e^{-t^{2} / 2} d t\right)^{b}
$$

One can verify that $f($.$) is a strictly concave function for$ $b \in\{1,2,3,4,5\}$. In the simulations below we use $b=4$. The fading channel is taken to be Rayleigh [20], so that $h_{k}$ is exponentially distributed with p.d.f.

$$
p\left(h_{k}\right)=\frac{1}{\bar{h}} \exp \left(-h_{k} / \bar{h}\right), h_{k} \geq 0
$$

with $\bar{h}$ being its mean. Here we will use $\bar{h}=1$. In solving the Bellman equation (5) we use 50 discretization points for each of the quantities $P_{k}, h_{k}, u_{k}$.

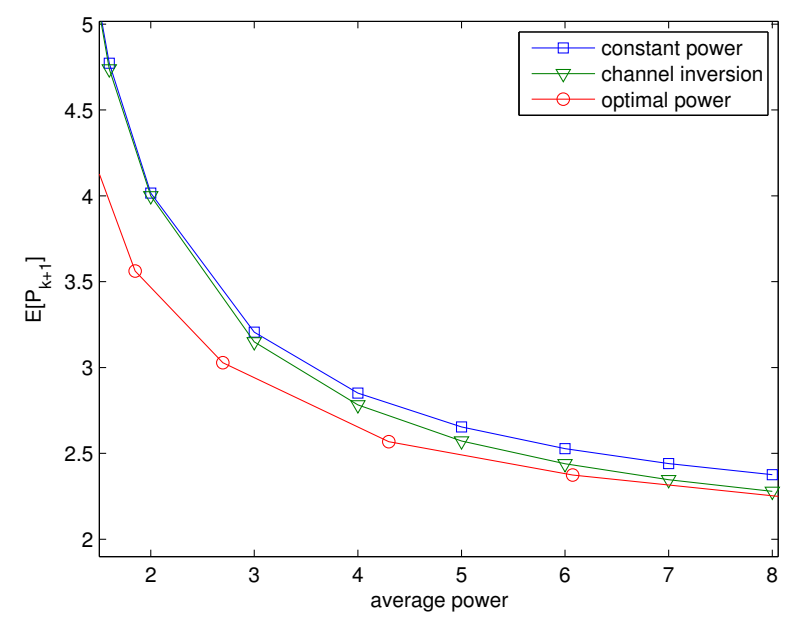

Fig. 2. Average transmit power vs expected error covariance

In Fig. 2 we plot the average transmit power vs expected error covariance tradeoff, for the cases of optimal power allocation of Section III, and the constant power allocation and truncated channel inversion policies of Section IV. We see that optimal power allocation has significant performance gains over the simpler suboptimal policies of Section IV for low average transmit powers, with the performance of the constant power allocation and channel inversion policies being almost identical. However, for higher average transmit powers, the truncated channel inversion policy has performance approaching that of the the optimal power allocation policy.

In Fig. 3 we show a 3D plot of the optimal power allocation $u_{k}$ as a function of $P_{k}$ and $h_{k}$ given by solving the MDP (5), for parameters $\beta=1$ and $u_{\max }=10$. With 
these parameters, the average power is around 2.05 and $\mathbb{E}\left[P_{k+1}\right]$ is around 3.36 . In Fig. 4 we further plot a single

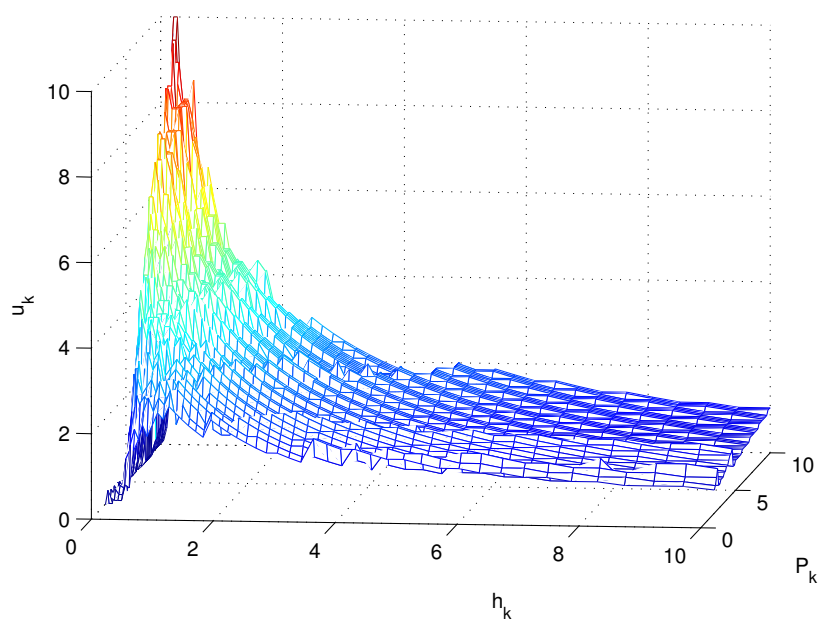

Fig. 3. Optimal power allocations

simulation run of $P_{k}$ and $h_{k}$, together with the corresponding optimal power allocations $u_{k}$ We can see that in the optimal
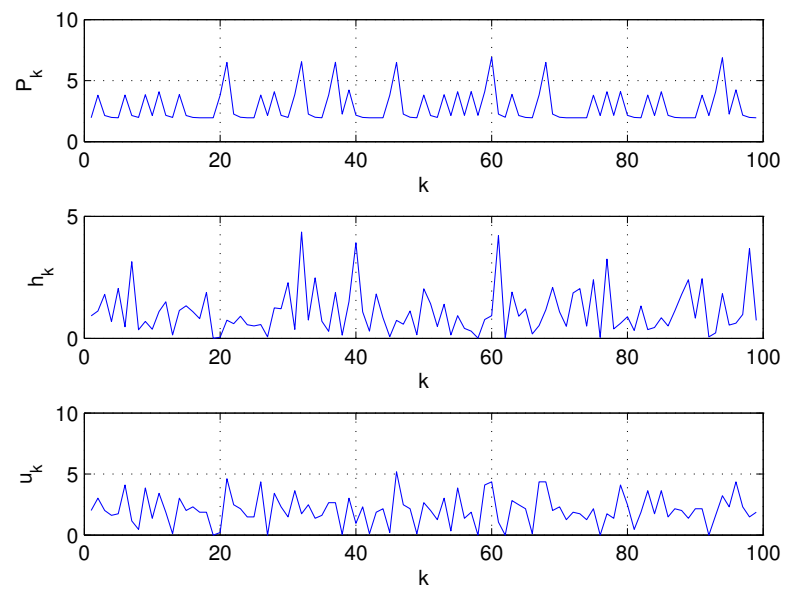

Fig. 4. Optimal power allocations

power allocation scheme, the allocated powers will depend on both the current channel gain $h_{k}$ and error covariance $P_{k}$. The allocated power $u_{k}$ tends to be higher when the error covariance $P_{k}$ is larger, provided the corresponding channel gain $h_{k}$ is not too small.

\section{CONCLUSION}

We have studied the problem of minimizing the trace of the expected error covariance subject to a average power constraint, for Kalman filtering with random packet losses. Simpler suboptimal power allocation policies such as a constant power allocation policy and a truncated channel inversion policy policies have also been considered. Numerical studies suggest that for low average transmit powers optimal power allocation significantly outperforms the suboptimal policies, while for higher average transmit powers the performance of the truncated channel inversion policy approaches the performance of the optimal policy. Future work will include the study of systems with multiple sensors and the consideration of other fading models such as the Markovian fading channel model of [21].

\section{APPENDIX}

\section{A. Proof of Lemma 2}

Proof: The proof uses similar ideas to the proof of Proposition 3.1 in [28]. The decreasing property follows from the relation

$$
\begin{aligned}
& \mathbb{E}\left[P_{k+1}\right]=\mathbb{E}\left[P_{k+1} \mid P_{k}, h_{k}, u_{k}\right] \\
& =\mathbb{E}\left[A P_{k} A^{T}+Q-f\left(h_{k} u_{k}\right) A P_{k} C^{T}\left(C P_{k} C^{T}+R\right)^{-1} C P_{k} A^{T}\right]
\end{aligned}
$$

and the assumption that $f($.$) is an increasing function.$

For the proof of convexity, let $u^{1}$ and $u^{2}$ be two average transmit powers, $u^{1} \neq u^{2}$, with $P^{*}\left(u^{1}\right)$ and $P^{*}\left(u^{2}\right)$ the corresponding traces of the expected error covariances. We want to show that

$$
P^{*}\left(\lambda u^{1}+(1-\lambda) u^{2}\right)<\lambda P^{*}\left(u^{1}\right)+(1-\lambda) P^{*}\left(u^{2}\right), \forall \lambda \in(0,1)
$$

Let $\left\{u_{k}^{1}\left(P_{k}, h_{k}\right)\right\}$ be the optimal power allocation policy that achieves $P^{*}\left(u^{1}\right)$, and $\left\{u_{k}^{2}\left(P_{k}, h_{k}\right)\right\}$ be the optimal power allocation policy that achieves $P^{*}\left(u^{2}\right)$. Define a new policy $\left\{u_{k}^{\lambda}\left(P_{k}, h_{k}\right)\right\}$ such that

$$
u_{k}^{\lambda}\left(P_{k}, h_{k}\right)=\lambda u_{k}^{1}\left(P_{k}, h_{k}\right)+(1-\lambda) u_{k}^{2}\left(P_{k}, h_{k}\right), \forall P_{k}, h_{k}
$$

We will first show that for a given $P_{k}$, we have:

$$
\begin{aligned}
& \text { 1) } \mathbb{E}\left[u_{k}^{\lambda} \mid P_{k}\right] \leq \lambda \mathbb{E}\left[u_{k}^{1} \mid P_{k}\right]+(1-\lambda) \mathbb{E}\left[u_{k}^{2} \mid P_{k}\right], \text { and } \\
& 2) \mathbb{E}\left[\operatorname{tr}\left(P_{k+1}^{\lambda}\right) \mid P_{k}\right] \\
& \quad<\lambda \mathbb{E}\left[\operatorname{tr}\left(P_{k+1}^{1}\right) \mid P_{k}\right]+(1-\lambda) \mathbb{E}\left[\operatorname{tr}\left(P_{k+1}^{2}\right) \mid P_{k}\right],
\end{aligned}
$$

where $P_{k+1}^{j}$ is the value of $P_{k+1}$ that follows from using policy $\left\{u_{k}^{j}().\right\}$, for $j=1,2, \lambda$ respectively. For 1 ), this clearly follows from the definition of $u_{k}^{\lambda}$. For 2), we have

$$
\begin{aligned}
& \mathbb{E}\left[\operatorname{tr}\left(P_{k+1}^{\lambda}\right) \mid P_{k}\right] \\
& =\int\left(\operatorname{tr}\left(A P_{k} A^{T}+Q\right)\right. \\
& \left.\quad-f\left(h_{k} u_{k}^{\lambda}\right) \operatorname{tr}\left(A P_{k} C^{T}\left(C P_{k} C^{T}+R\right)^{-1} C P_{k} A^{T}\right)\right) F\left(d h_{k}\right) \\
& <\int\left(\operatorname{tr}\left(A P_{k} A^{T}+Q\right)\right. \\
& \quad-\left(\lambda f\left(h_{k} u_{k}^{1}\right)+(1-\lambda) f\left(h_{k} u_{k}^{2}\right)\right) \\
& \left.\quad \times \operatorname{tr}\left(A P_{k} C^{T}\left(C P_{k} C^{T}+R\right)^{-1} C P_{k} A^{T}\right)\right) F\left(d h_{k}\right) \\
& =\lambda \mathbb{E}\left[\operatorname{tr}\left(P_{k+1}^{1}\right) \mid P_{k}\right]+(1-\lambda) \mathbb{E}\left[\operatorname{tr}\left(P_{k+1}^{2}\right) \mid P_{k}\right]
\end{aligned}
$$

where the inequality comes from the strict concavity of $f($.$) .$ 
From 1) and 2), we have

$$
\begin{aligned}
\lim _{K \rightarrow \infty} & \frac{1}{K} \sum_{k=1}^{K} \mathbb{E}\left[u_{k}^{\lambda}\right]=\lim _{K \rightarrow \infty} \frac{1}{K} \sum_{k=1}^{K} \mathbb{E}\left[\mathbb{E}\left[u_{k}^{\lambda} \mid P_{k}\right]\right] \\
& \leq \lim _{K \rightarrow \infty} \frac{1}{K} \sum_{k=1}^{K} \mathbb{E}\left[\lambda \mathbb{E}\left[u_{k}^{1} \mid P_{k}\right]+(1-\lambda) \mathbb{E}\left[u_{k}^{2} \mid P_{k}\right]\right] \\
& =\lambda u^{1}+(1-\lambda) u^{2}
\end{aligned}
$$

and

$$
\begin{aligned}
& \lim _{K \rightarrow \infty} \frac{1}{K} \sum_{k=1}^{K} \mathbb{E}\left[\operatorname{tr}\left(P_{k+1}^{\lambda}\right)\right]=\lim _{K \rightarrow \infty} \frac{1}{K} \sum_{k=1}^{K} \mathbb{E}\left[\mathbb{E}\left[\operatorname{tr}\left(P_{k+1}^{\lambda}\right) \mid P_{k}\right]\right] \\
&<\lim _{K \rightarrow \infty} \frac{1}{K} \sum_{k=1}^{K} \mathbb{E}\left[\lambda \mathbb{E}\left[\operatorname{tr}\left(P_{k+1}^{1}\right) \mid P_{k}\right]\right. \\
&\left.+(1-\lambda) \mathbb{E}\left[\operatorname{tr}\left(P_{k+1}^{2}\right) \mid P_{k}\right]\right] \\
&=\lambda P^{*}\left(u^{1}\right)+(1-\lambda) P^{*}\left(u^{2}\right) .
\end{aligned}
$$

By the definition of $P^{*}(u)$ being the minimum expected error covariance such that the average transmit power is less than or equal to $u$, we then have $P^{*}\left(\lambda u^{1}+(1-\lambda) u^{2}\right) \leq$ $\frac{1}{K} \sum_{k=1}^{K} \mathbb{E}\left[\operatorname{tr}\left(P_{k+1}^{\lambda}\right)\right]<\lambda P^{*}\left(u^{1}\right)+(1-\lambda) P^{*}\left(u^{2}\right)$.

\section{REFERENCES}

[1] B. Sinopoli, L. Schenato, M. Franceschetti, K. Poolla, M. I. Jordan, and S. S. Sastry, "Kalman filtering with intermittent observations," IEEE Trans. Autom. Control, vol. 49, no. 9, pp. 1453-1464, September 2004.

[2] X. Liu and A. J. Goldsmith, "Kalman filtering with partial observation losses," in Proc. IEEE Conf. Decision and Control, Bahamas, Dec. 2004, pp. 1413-1418.

[3] V. Gupta, N. C. Martins, and J. S. Baras, "Optimal output feedback control using two remote sensors over erasure channels," IEEE Trans. Autom. Control, vol. 54, no. 7, pp. 1463-1476, Jul. 2009.

[4] K. Plarre and F. Bullo, "On Kalman filtering for detectable systems with intermittent observations," IEEE Trans. Autom. Control, vol. 54, no. 2, pp. 386-390, Feb. 2009.

[5] Y. Mo and B. Sinopoli, "A characterization of the critical value for Kalman filtering with intermittent observations," in Proc. IEEE Conf. Decision and Control, Cancun, Mexico, Dec. 2008.

[6] M. Epstein, L. Shi, A. Tiwari, and R. M. Murray, "Probabilistic performance of state estimation across a lossy network," Automatica, vol. 44, pp. 3046-3053, Dec. 2008.

[7] L. Shi, M. Epstein, and R. M. Murray, "Kalman filtering over a packetdropping network: A probabilistic perspective," IEEE Trans. Autom. Control, vol. 55, no. 3, pp. 594-604, Mar. 2010.

[8] Y. Xu and J. P. Hespanha, "Estimation under uncontrolled and controlled communications in networked control systems," in Proc. IEEE Conf. Decision and Control, Seville, Spain, December 2005, pp. 842847.

[9] L. Schenato, "Optimal estimation in networked control systems subject to random delay and packet drop," IEEE Trans. Autom. Control, vol. 53, no. 5, pp. 1311-1317, Jun. 2008.

[10] M. Huang and S. Dey, "Stability of Kalman filtering with Markovian packet losses," Automatica, vol. 43, pp. 598-607, 2007.

[11] L. Xie and L. Xie, "Stability of a random Riccati equation with Markovian binary switching," IEEE Trans. Autom. Control, vol. 53, no. 7, pp. 1759-1764, Aug. 2008.

[12] K. You, M. Fu, and L. Xie, "Mean square stability for Kalman filtering with Markovian packet losses," Automatica, vol. 47, no. 12, pp. 12471257, Dec. 2011.

[13] Y. Mostofi and R. M. Murray, "On dropping noisy packets in Kalman filtering over a wireless fading channel," in Proc. American Control Conf., Portland, OR, Jun. 2005, pp. 4596-4600.

[14] S. Dey, A. S. Leong, and J. S. Evans, "Kalman filtering with faded measurements," Automatica, vol. 45, no. 10, pp. 2223-2233, Oct. 2009.
[15] A. S. Leong, S. Dey, G. N. Nair, and P. Sharma, "Power allocation for outage minimization in state estimation over fading channels," IEEE Trans. Signal Process., vol. 59, no. 7, pp. 3382-3397, Jul. 2011.

[16] D. E. Quevedo, A. Ahlén, and J. Østergaard, "Energy efficient state estimation with wireless sensors through the use of predictive power control and coding," IEEE Trans. Signal Process., vol. 58, no. 9, pp. 4811-4823, Sep. 2010.

[17] D. E. Quevedo, A. Ahlén, A. S. Leong, and S. Dey, "On Kalman filtering over fading wireless channels with controlled transmission powers," Automatica, vol. 48, no. 7, pp. 1306 - 1316, Jul. 2012.

[18] J. G. Proakis, Digital Communications, 4th ed. New York: McGrawHill, 2001.

[19] G. Caire, G. Taricco, and E. Biglieri, "Optimum power control over fading channels," IEEE Trans. Inf. Theory, vol. 45, no. 5, pp. 14681489, Jul. 1999.

[20] T. S. Rappaport, Wireless Communications: Principles and Practice, 2nd ed. New Jersey: Prentice Hall, 2002.

[21] D. E. Quevedo, A. Ahlén, and K. H. Johannson, "Stability of state estimation over sensor networks with Markovian fading channels," in Proc. IFAC World Congress, Milan, Italy, Aug. 2011.

[22] O. Hernández-Lerma, J. González-Hernández, and R. R. LópezMartínez, "Constrained average cost Markov control processes in Borel spaces," SIAM J. Contr. and Optim., vol. 42, no. 2, pp. 442-468, 2003.

[23] N. Ghasemi and S. Dey, "Power-efficient dynamic quantization for multisensor HMM state estimation over fading channels," in Proc. ISCCSP, Malta, Mar. 2008, pp. 1553-1558.

[24] O. Hernández-Lerma and J. B. Lasserre, Discrete-Time Markov Control Processes: Basic Optimality Criteria. New York: Springer-Verlag, 1996.

[25] D. P. Bertsekas, Dynamic Programming and Optimal Control, Volumes I and II, 2nd ed. Belmont, Massachusetts: Athena Scientific, 2000.

[26] H. Yu and D. P. Bertsekas, "Discretized approximations for POMDP with average cost," in Proc. 20th Conference on Uncertainty in Artifical Intelligence, Banff, Canada, 2004, pp. 619-627.

[27] S. Boyd and L. Vandenberghe, Convex Optimization. Cambridge, Great Britain: Cambridge University Press, 2004.

[28] R. A. Berry and R. G. Gallager, "Communication over fading channels with delay constraints," IEEE Trans. Inf. Theory, vol. 48, no. 5, pp. 1135-1149, May 2002.

[29] A. J. Goldsmith and P. P. Varaiya, "Capacity of fading channels with channel side information," IEEE Trans. Inf. Theory, vol. 43, no. 6, pp. 1986-1992, Nov. 1997. 